\title{
Intrinsic Properties of Metamaterial Slab in Wireless Power Transfer for Efficiency Improvement of Wireless Communication Systems
}

\author{
Abdul Syed $\dagger^{+*}$ and Professor Kanti Prasad ${ }^{\ddagger}$ \\ †Department of Electrical and Computer Engineering (ECE), Francis College of Engg. (FCE), UMass. Lowell, USA \\ †Electronics and Communication Engineering (ECE) Department, Muffakham Jah College of Engineering and Technology (MJCET), Osmania \\ University, Hyderabad, India
}

Received 11 Nov 2017, Accepted 10 Jan 2018, Available online 12 Feb 2018, Vol.8, No.1 (Jan/Feb 2018)

\begin{abstract}
Metamaterial (MTM) slabs, realized using Split Ring Resonators (SRR's) to enhance efficiency of Wireless Power Transfer (WPT) systems is discussed here. A detailed study of intrinsic properties obtained by simulation of the actual MTM slab is presented. An improvement in the wavelength to unit cell size of 813, at a low operating frequency of $5680 \mathrm{kHz}$ is shown. Forward transmission coefficients $\left(S_{21}\right)$ for with and without MTM slab cases are compared. In this low frequency regime, with simulated S-parameters an efficiency of $\sim 68 \%$ is achieved by placing the MTM slab closer to receiver coil than the transmitter coil.
\end{abstract}

Keywords: Wireless Power Transfer (WPT), Split Ring Resonator (SRR), Magnetic Resonance, Metamaterial (MTM), Bandpass Filter (BPF), Smartphone.

\section{Introduction}

WPT technologies based on near-field coupling are leading the research of magnetic resonance coupling over inductive coupling mode theory, for the development of metamaterials (E. Waffenschmidt and T. Staring, 2009). The later suffers the drawbacks of having closer coils proximity for high efficiency needs, difficult resonance matching conditions that are investigated in (E. Waffenschmidt and T. Staring, 2009). For artificially engineered metamaterials, their complex material parameters such as permittivity $(\varepsilon)$ and permeability $(\mu)$ play an important role in manipulation of intrinsic material properties. Measurement of which shall be an important aspect of their characterization useful in designing communication systems having dual features of compactness and WPT.

Various efforts in the miniaturization of resonant inclusions for realizing MTM's have been presented in (F. Aznar, and J. Bonache et al, 2008; F. Aznar, and J. García-Garcı'a et al, 2008; F. Bilotti et al, 2007; K. B. Alici et al, 2007), where very small electrical sizes (below $\lambda / 100$ ), for SRR (resonant elements) compared to the wavelength $(\lambda)$ is shown in (F. Aznar, and J. Bonache et al, 2008). In (F. Bilotti et al, 2007) resonant inclusions are deliberated MSRR's (multiple SRR's) while greater miniaturization rate at $\mathrm{GHz}$ frequencies is detailed in (F. Aznar, and J. Garcl'a-Garcl'a et al,

*Corresponding author's ORCID ID: 0000-0002-9641-881X DOI: https://doi.org/10.14741/ijcet/v.8.1.22
2008). Since SRR's are electro-magnetic microstructures for achieving negative values of effective $\mu$, that have applications in advanced imaging (M. J. Freire and R. Marques, 2005) to compact antennas (K. Buell et al, 2006), therefore having an effective medium ratio $(\lambda / a)$ much larger is desired as summarized in (K. B. Alici et al, 2007).

The size of unit cell reported in (B. Wang et al, 2011) was $6.5 \mathrm{~cm}$ and planar spirals with a spacing of $1.5 \mathrm{~cm}$, between each neighboring turns resulted in a wavelength to unit cell ratio of 170 . A summary of the progress in the work of (B. Wang et al, 2011) is given in (B. Wang et al, 2013), where the ratio $(\lambda / \mathrm{a})$ value is 170 for the operating frequency of $27.12 \mathrm{MHz}$. Also in 2013, (Y. Fan et al, 2013) demonstrated using double spirals MTM structure, the ratio $(\lambda / \mathrm{a})$ value of 253 at a frequency of $14.6 \mathrm{MHz}$ for coils separated by $14 \mathrm{~cm}$.

These results are not feasible for cases where a large number of unit cells (resonant SRR elements) are to be placed in a confined area such as in wireless handsets, to have compact MTM slab configurations. Primary technique used to obtain composite slabs of MTM's is through miniaturization agenda of unit cells acting as resonant inclusions, discussed and outlined in ( $F$ Aznar, and J. Bonache et al, 2008; F. Aznar, and J. Garci'a-Garci'a et al, 2008; F. Bilotti et al, 2007; K. B. Alici et al, 2007).

In 2014, (A. Rajagopalan et al, 2014) demonstrated a spiral structure operating at $23.7 \mathrm{MHz}$ using $1.6 \mathrm{~cm}$ compact unit cells, where a ratio value of 744 was reported. However the setup in (A. Rajagopalan et al, 
2014), at an operating frequency of $26.65 \mathrm{MHz}$ provided a maximum efficiency of only $18.23 \%$, despite of using MTM where a small separation in coils distance of $1 \mathrm{~cm}$ is considered, which is deemed low for real time usage.

The results of (A. Rajagopalan et al, 2014; A. K. RamRakhyani et al, 2011), though are given for shortrange telemetry systems such as implantable biomedical devices, but are not desirable for applications where a larger coils separation distance along with reduced operating frequency and better efficiency of WPT system is needed (E. Waffenschmidt and T. Staring, 2009; B. Wang et al, 2013; Y. Fan et al, 2013).

We demonstrate improvement in $(\lambda / a)$ ratio value of MTM slab realized using SRR's, at a low frequency of $5680 \mathrm{kHz}$, without compromising the coils separation distance of $50 \mathrm{~cm}$ presented in (B. Wang et al, 2013), along with improvement in the overall system efficiency. This can lead in the development of a different class of cost-effective MTM's, having greater number of SRR unit cell inclusions per slab, at lower frequencies. Such a slab also minimizes use of multiple MTM slabs in (Y. Fan et al, 2013), along with providing safe use for human beings in consumer wireless such as smartphones and bio-medical applications along with those mentioned in (E. Waffenschmidt and T. Staring, 2009; A. K. RamRakhyani et al, 2011).

\section{Wireless Power Transfer via Magnetic Resonance (Near Field Coupling)}

A typical WPT model consists of two coil resonators, one for transmission and another for reception along with MTM slab, as shown in Fig.1. A slab of MTM unit cell as shown in Fig.2 (a), is simulated using the finite integration technique, and perfect boundary approximations in CST (CST, 2014). For simulation purposes, a copper substrate of thickness $11.57 \mathrm{~mm}$ having relative permittivity $\varepsilon_{\mathrm{r}}=3.55$ and conductivity $\sigma=5.8 \times 10^{7} \mathrm{~S} / \mathrm{m}$ is taken. Copper foil is $0.07 \mathrm{~mm}$ thick. Discrete edge ports ( $50 \Omega$ each), are used to simulate the loop coils which act as lumped network element sources (basic LC equivalent resonators).

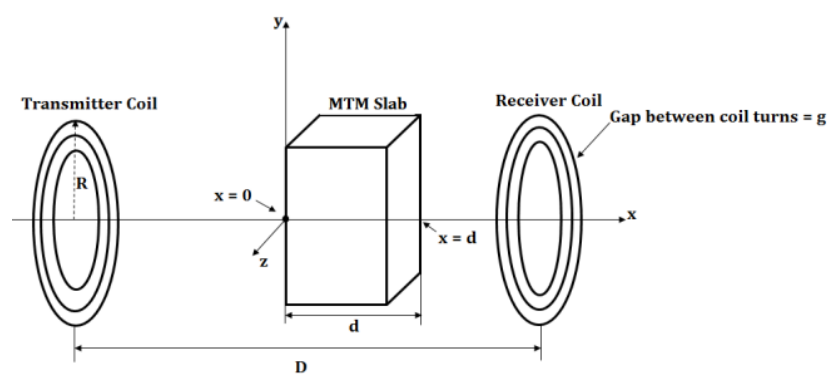

Fig.1 Configuration of WPT communications system with MTM slab consisting of Coils (SRR's)

A small amount of loss tangent delta $(\tan \delta=0.0027$ Rogers corporation (RO) 4003C material) is included in obtaining simulations, to consider a practical MTM slab, $1.5 \mathrm{~mm}$ thick. The length of square ring ' $\mathrm{L}$ ' is 61 $\mathrm{mm}$, while the distance between the resonant coils ' $\mathrm{D}$ ' is $500 \mathrm{~mm}$ and the width of each unit cells ' $a$ ' is $65 \mathrm{~mm}$. Copper strip width ' $W$ ' of $3 \mathrm{~mm}$ with $1 \mathrm{~mm}$ spacing between each neighboring strips ' $S$ ' is considered for the square ring dimensions shown in Fig.2 (a). Planar spiral copper coil resonators having large/outer radius $\mathrm{R}=400 \mathrm{~mm}$, with gap ' $\mathrm{g}$ ' between each coil turns equal to $1 \mathrm{~mm}$ is used for performing simulations.

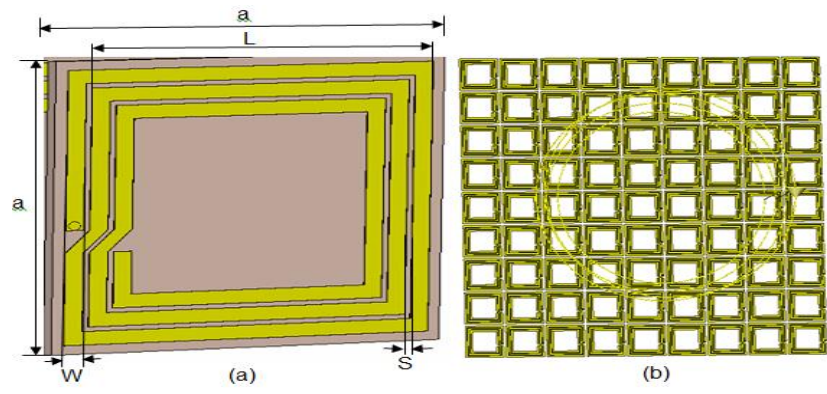

Fig.2 (a) Single square ring - Unit cell/SRR (J. B. Pendry et al, 1999)

(b) MTM slab realized using SRR's and CST modeling software (J. B. Pendry et al, 1999;

CST, 2014)

The basic material properties that are intrinsic to magneto-electric coupling where the electric field $\mathrm{E}$ causes magnetic polarization and vice-versa, are permeability $(\mu)$, permittivity $(\varepsilon)$. Using the Smith's method (D. R. Smith et al, 2002), the effective properties of the MTM slab are determined after simulating the single unit cell, composed of a single square ring [such as a simple SRR (J. B. Pendry et al, 1999), made of copper], having geometric parameters as defined earlier with appropriate boundary conditions. Then, the effective material parameters $\mu_{\text {eff }}, \varepsilon_{\text {eff }}$ are extracted using the simulated $S$ parameters and Lorentz-Drude material models. The S - parameters are as shown in Fig.3, while the extracted effective material properties are shown in Fig.4 and Fig.5.

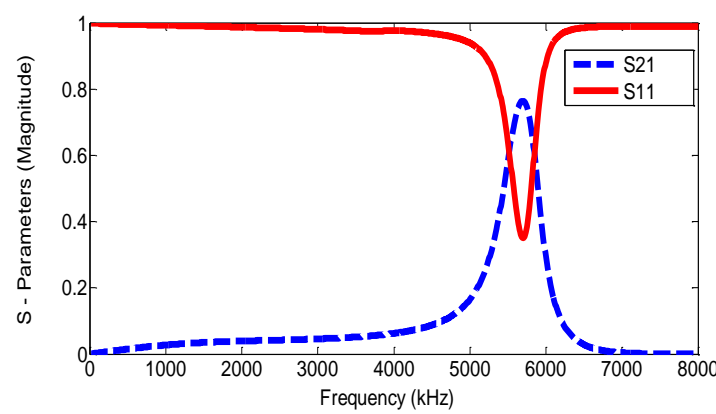

Fig.3 S - parameters from simulations with MTM slab at $300 \mathrm{~mm}$ from the transmitter side

Knowing these intrinsic properties, the values of effective impedance $Z_{\text {eff }}$ and effective refractive index $\mathrm{n}_{\text {eff }}$ can be computed using Smith's method (D. R. Smith 
et al, 2002). For low frequencies compared to the dimensions of discrete ports, the dielectric losses, reflections are small resulting in successful Sparameter calculations, using CST's frequency domain solver. Also the $S_{11}, S_{21}$ shown in Fig.3, for multiple cells of MTM match the $\mu_{\text {eff }}, \varepsilon_{\text {eff }}$ retrieved for a unit cell, implying effective homogeneity for MTM slab. Thus a unit cell size, which is 813 times smaller than the operating wavelength of $52.81 \mathrm{~m}$ is realized.

As seen in Fig.4, SRR inclusions on MTM slab, that were shown in Fig.2 (b), with slab being centered between the two coils, produces a frequency region where the real part of $\mu_{\text {eff }}$ value is negative (D. R. Smith et al, 2002). Very large negative real $\mu_{\text {eff }}$ value of MTM slab causes evanescent amplification, by guiding the magnetic flux which is essentially causing the efficiency enhancement, based on LC resonance effect.

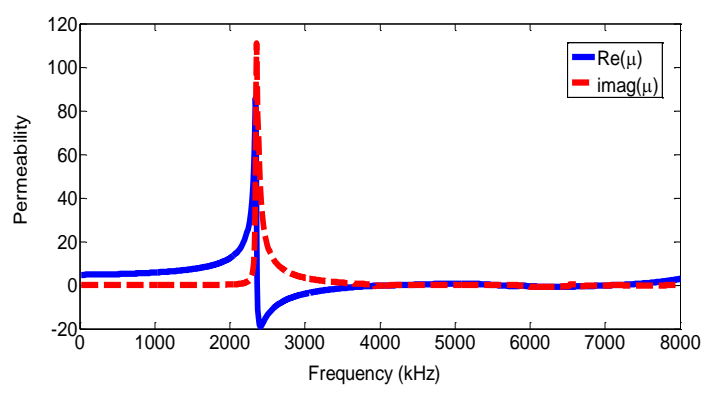

Fig.4 Effective permeability extracted using method in (D. R. Smith et al, 2002)

By studying the properties of unit cell of Fig.2 (a), we are affirming its true likeness to the MTM slab attributes, whose realization is shown in Fig.2 (b). Because the practical slab has an periodic arrangement of homogeneous scattering elements, the unit cell dimensions choosen are thus much smaller than the guided wavelength of the MTM slab itself.

In Fig.5, exhibiting resonance behaviour at various frequencies, is satisfying two basic conditions where real part of $\varepsilon_{\text {eff }}$ is negative and the wavelength is large in comparision to dimensions. Due to interaction of mobile electrons with the electromagnetic radiations, the dispersion relations given in (D. R. Smith et al, 2002), are fulfilled mostly near the plasma frequencies. For this reason low frequency has been a viable option in wireless communication and current Long Term Evolution (LTE) networks.

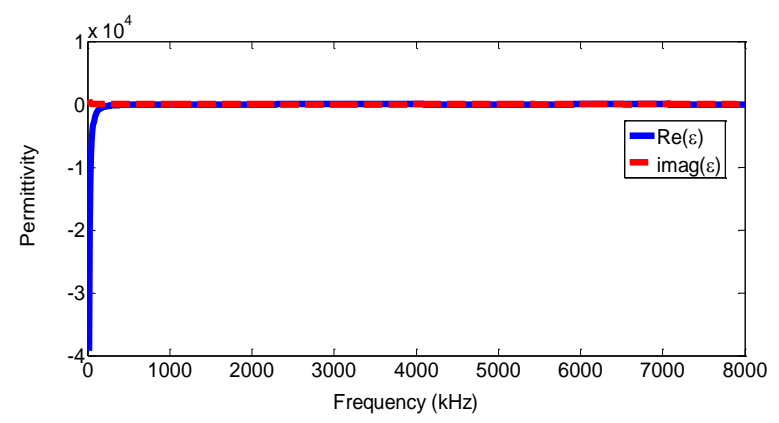

Fig.5 Effective permittivity extracted using method in (D. R. Smith et al, 2002)
In Fig.1, the radius of the coil $\mathrm{R}$ has influence on inductance $\mathrm{L}$, capacitance $\mathrm{C}$, frequency and efficiency, with radius $\mathrm{R}$ having inverse relation to resonant frequency $f_{0}$, governed by $f_{0}=\frac{1}{2 \pi \sqrt{\mathrm{LC}}}$. Also $\mathrm{L}, \mathrm{C}$ increases as $\mathrm{R}$ increases, due to increase in the length of wire (S. Konno et al, 2013; N. Gvozdenovic et al, 2013). However as the gap ' $g$ ' between coils decreases, the coupling coefficient increases, increasing the system efficiency (N. Gvozdenovic et al, 2013).

It is evident from Fig.3, that around resonance $S_{21}$ values are larger and taking into account the non-zero $S_{11}$ values, the peak efficiency $\eta$ of a two coil system is given by (S. V. Georgakopoulos et al, 2011).

$\eta=\frac{\left|S_{21}\right|^{2}}{\left(1-\left|S_{11}\right|^{2}\right)}$

An efficiency value of $68.35 \%$ is calculated using equation (1), by considering the S-parameters shown in Fig.3 that were obtained by keeping the MTM slab at $300 \mathrm{~mm}$ from the transmitter coil. The resonance frequency with slab closer to receiver coil, is thus shifted to $5680 \mathrm{kHz}$ while the coils separation distance was still kept at $500 \mathrm{~mm}$. A simulated value of wavelength to unit cell size of 813 and an improvement in the efficiency value of $\sim 68 \%$ is achieved, predominantly due to increase in mutual coupling.

\section{Evanescent Waves amplified most using MTM Slab structure with SRR inclusions}

As the $S_{11}$ values in Fig. 3, are small at resonance frequency, we can consider efficiency essentially to be $\approx\left|S_{21}\right|^{2}$ (B. Wang et al, 2011). By keeping MTM slab at different positions between the two coils depicted in Fig.1, the efficiency variations w.r.t distance are shown in Fig.6. In this case the peak efficiency is $\approx 60 \%$.

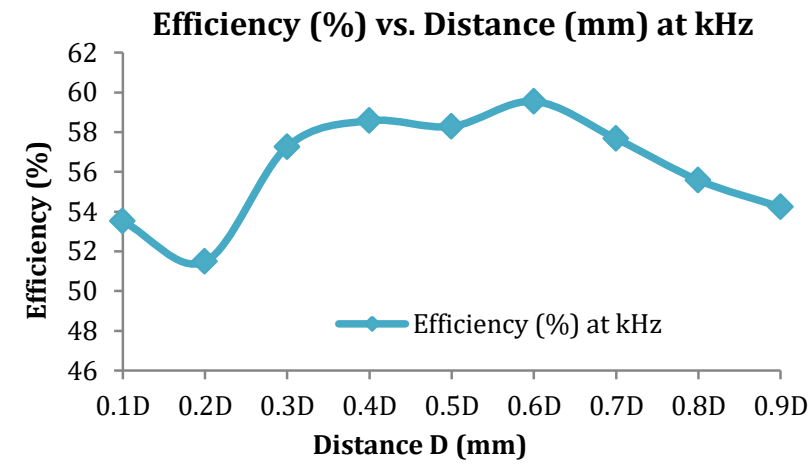

Fig.6 Efficiency $\eta\left[\eta \approx\left|S_{21}\right|^{2}\right.$ (B. Wang et al, 2011)] versus distance $D(D=500 \mathrm{~mm})$, with MTM slab.

Fig.7, shows the comparison of efficiencies for two different WPT experimental cases involving MTM slab's presence and MTM slab's absence. The MTM slab is centered between the transmitter and receiver coils while the coils separation is left unchanged in both these WPT experiments. It can be seen clearly that the case with MTM slab contribute to higher efficiency than the case involving free-space only. 


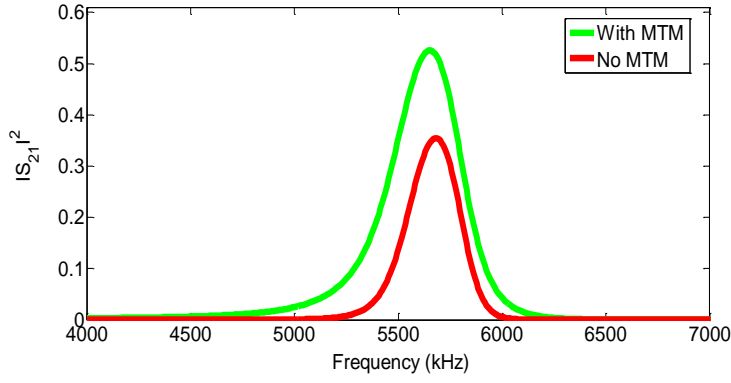

Fig.7 $\left|S_{21}\right|^{2}$ (Efficiency) versus frequency for with MTM slab and without MTM slab

Table 1 Summary of geometric parameters used for MTM based WPT communication system

\begin{tabular}{|c|c|c|c|}
\hline S. No & Parameter & Value $(\mathrm{mm})$ & Description \\
\hline 1 & $\mathrm{~L}$ & 61 & Square ring length (SRR) \\
\hline 2 & $\mathrm{R}$ & 400 & $\begin{array}{c}\text { Large/Outer Radius of } \\
\text { resonators }\end{array}$ \\
\hline 3 & $\mathrm{~S}$ & 1 & $\begin{array}{c}\text { Spacing between each } \\
\text { neighboring strips }\end{array}$ \\
\hline 4 & $\mathrm{~d}$ & 1.5 & MTM slab thickness \\
\hline 5 & $\mathrm{~W}$ & 3 & Copper strip width \\
\hline 6 & $\mathrm{a}$ & 65 & $\begin{array}{c}\text { Period of Unit Cells } \\
\text { (width) }\end{array}$ \\
\hline 7 & $\mathrm{D}$ & 500 & $\begin{array}{c}\text { Distance between } \\
\text { transmitter, receiver coils }\end{array}$ \\
\hline 8 & $\mathrm{~g}$ & 1 & $\begin{array}{c}\text { Gap between each coil } \\
\text { turns of resonators }\end{array}$ \\
\hline
\end{tabular}

\section{Conclusions}

A numerical study of different intrinsic properties, followed by manipulation of geometric parameters in realizing unit cell SRR was performed. We achieved improvement in the wavelength to unit cell size ratio at a low operating frequency of $5680 \mathrm{kHz}$. Finally efficiency enhancement for the MTM based WPT system to $\sim 68 \%$, using the realized SRR having $\lambda / \mathrm{a}$ value of 813 is presented in this paper.

This study shall lead to numerous applications in consumer wireless and smartphones technology design specifications including efficient WPT. Demand of simultaneous compact shared antennas capabilities with BPF requirements in the frontend modules at LTE bands can be addressed by incorporating these MTM features in next Generation wireless communications.

\section{Acknowledgment}

This is a university directed independent research work. The author thanks the FCE/UMass LowellDepartment of ECE for providing the stipend, modeling software, and departmental resources to perform this work. Thanks are also due to the ECE doctoral program coordinator at FCE/UMass Lowell, for providing deep insights and expertise of the WPT, MTM areas.

\section{References}

E. Waffenschmidt, and T. Staring (2009), Limitation of inductive power transfer for consumer applications, 13 th European Conference on Power Electronics and Applications, EPE 09, pp. 1-10.

F. Aznar, J. Bonache, F. Martin, E. Ozbay, K. Alici, F. Bilotti, S. Tricarico, L. Vegni, J. Baena, L. Jelinek, and R. Marques (2008), Miniaturization and characterization of metamaterial resonant particles, $38^{\text {th }}$ European Conference on Microwave Conference, EuMC 08, pp. 269-272.

F. Aznar, J. Garci'a-Garci'a, M. Gil, J. Bonache, and F. Martín (2008), Strategies for the miniaturization of metamaterial resonators, Microwave and Optical Tech. Letters, vol. 50, no. 5.

F. Bilotti, A. Toscano, and L. Vegni (2007), Design of spiral and multiple split-ring resonators for the realization of miniaturized metamaterial samples, IEEE Trans. on Antennas and Propag., vol. 55, no. 8, pp. 2258-2267.

K. B. Alici, F. Bilotti, L. Vegni, and E. Ozbay (2007), Miniaturized negative permeability materials, Applied Physics Letters, vol. 91, no. 7, pp. 071 121-071 121-3.

M. J. Freire, and R. Marques (2005), Appl. Phys. Lett. 86, 182505.

K. Buell, H. Mosallaei, and K. Sarabandi (2006), A substrate for small patch antennas providing tunable miniaturization factors, IEEE Trans. Microw. Theory Tech., vol. MTT-54, pp. 135-146.

B. Wang, K. H. Teo, T. Nishino, W. Yerazunis, J. Barnwell, and J. Zhang (2011), Experiments on wireless power transfer with metamaterials, Appl. Phys. Lett., vol. 98, 254101.

B. Wang, K. H. Teo, and W. Yerazunis (2013), Wireless Power Transfer: Metamaterials and Array of Coupled Resonators, Proceedings of the IEEE, vol. 101, no. 6.

Y. Fan, L. Li, S. Yu, C. Zhu, and C. Liang (2013), Experimental study of efficient wireless power transfer system integrating with highly sub-wavelength metamaterials, Progress In Electromagnetics Research, vol. 141, pp. 769-784.

A. Rajagopalan, A. K. RamRakhyani, D. Schurig, and G. Lazzi (2014), Improving power transfer efficiency of short-range telemetry system using compact metamaterials, IEEE transactions on Microwave Theory and Techniques, vol. 62, no. 4, pp. 947-955.

A. K. RamRakhyani, S. Mirabbasi, and M. Chiao (2011), Design and optimization of resonance-based efficient wireless power delivery systems for biomedical implants, IEEE Transactions on Biomedical Circuits \& Systems, vol. 5, no.1, pp. 48-63.

J. B. Pendry, A. J. Holden, D. J. Robbins, and W. J. Stewart (1999), Magnetism from conductors and enhanced nonlinear phenomena, IEEE Trans. Microw. Theory Tech., vol. 47, pp. 2075- 2084

CST (2014), Computer Simulation Technology AG, Microwave Studio Suite 2014, CST 1998-2013, Darmstadt, Germany.

D. R. Smith, S. Schultz, P. Markoš, and C. M. Soukoulis (2002), Determination of effective permittivity and permeability of metamaterials from reflection and transmission coefficients, Phys. Rev. B 65, 195104

S. Konno, T. Yamamoto, and K. Koshiji (2013), Improvement of coupling coefficient by designing a spiral pattern formed on a printed circuit board, IEEE 978-1-4673-5010-5/13.

N. Gvozdenovic, L. W. Mayer, R. Prestros, C. F. Mecklenbr"auker, and A. L. Scholtz (2013), PEEC Modeling of circular spiral coils, Proceedings of the $43^{\text {rd }}$ European Microwave Conference, EuMA 2013, pp. 7-10.

S. V. Georgakopoulos, and O. Jonah (2011), Optimized wireless power transfer to RFID sensors via magnetic resonance, IEEE AP-S/URSI 11. 